\title{
Stability Analysis of Fuzzy Sliding Mode Controlled Switched Reluctance Motor Drives
}

\author{
C.C. Chan, Y.J. Zhan and K.T. Chau \\ Department of Electrical \& Electronic Engineering, \\ The University of Hong Kong, Pokfulam Road, Hong Kong
}

\begin{abstract}
This paper presents a small deviant dynamic-state model and the system transfer functions for SRM drive system under different control modes with fuzzy sliding mode controller. With the help of the system open-loop and closed-loop transfer functions, the operation performances of SRM drive system are discussed. From the characteristics of the transfer functions, it is concluded that the FSMC speed regulator can be regarded as an adaptive PI regulator. The proposed scheme is applied to a $4 \mathrm{~kW}$ SRM drive and the system performance is verified by computer simulation results.
\end{abstract}

\section{NOMENCLATURE}

$L \quad$ Phase inductance

$K \quad$ Profile slope of inductance

$\mathbf{i}=\left[i_{1}, \ldots, i_{n}\right]^{\mathrm{T}} \quad$ Phase current vector

$\mathbf{U}_{s}=\left[\mathrm{U}_{1}, \ldots, U_{n}\right]^{\mathrm{T}}$ Phase voltage vector

$\theta \quad$ Rotor position

$\theta_{o n}, \theta_{o f f} \quad$ On, off angle

$T_{e}, T_{l} \quad$ Motor torque, load torque

$\omega_{r} \quad$ Rotor speed

$B \quad$ Viscous friction coefficient

$J \quad$ Moment of inertia

\section{INTRODUCTION}

The switched reluctance motor (SRM) provides some definite advantages, namely the simple and reliable brushless construction, high efficiency over wide speed and torque ranges, and low manufacturing cost, which are very attractive for both industrial applications and electric propulsion.

Due to the highly nonlinear nature of the SRM, the stability analysis of its drive system is rarely involved by literature. However the system stability is a very important aspect in drive theory and practical application, especially to those systems which employ novel control methods. The research work is targeted at the stability analysis of SRM drive system with an advanced fuzzy sliding mode control (FSMC) strategy by means of its small deviant dynamic-state linearized model. To date, the fuzzy logic control (FLC) [1]-[4] and the sliding mode control (SMC)[5]-[9] have been two different research topics. The basic idea of combining these two methodologies together and formulating the scheme of FSMC [10]-[12],[14] is that the ap- pearance of nonidealities such that the sliding surface of sliding mode control system is no longer crisp. Therefore, the SMC is introduced to treat nonlinearities of SRM and FLC is used to reduce the control chattering.

\section{SMALL SIGNAL ANALYSIS FOR SRM DRIVE}

The SRM drive system model is established under the assumptions that there is no magnetic saturation and negligible mutual inductance and losses. Thus, the SRM drive model is described as:

$$
\left\{\begin{array}{l}
\frac{d \mathbf{i}}{d t}=-\mathbf{L}^{-1}(\theta) \cdot K \mathbf{i} \omega_{r}+L^{-1}(\theta) \mathbf{U}_{s} \\
\frac{d \omega_{r}}{d t}=\frac{T_{e}-T_{l}}{J}-\frac{B}{J} \omega_{r} \\
\frac{d \theta}{d t}=\omega_{r}
\end{array}\right.
$$

The corresponding speed error and the speed derivative are selected as state variables:

$\left\{\begin{array}{l}x_{1}=\omega_{r}-\omega_{r}^{*} \\ x_{2}=\dot{\omega}_{r}=-\frac{B}{J} \omega_{r}+\frac{1}{J} T_{e}-\frac{1}{J} T_{l}\end{array}\right.$

where $\omega_{r}^{*}$ is the speed reference. By differentiating (2) and taking $u=\dot{T}_{e}$ as control variable, it results:

$$
\left(\begin{array}{l}
\dot{x}_{1} \\
\dot{x}_{2}
\end{array}\right)=\left(\begin{array}{cc}
0 & 1 \\
0 & -\frac{B}{J}
\end{array}\right)\left(\begin{array}{l}
x_{1} \\
x_{2}
\end{array}\right)+\left(\begin{array}{c}
0 \\
\frac{1}{J}
\end{array}\right) u+\left(\begin{array}{c}
-\dot{\omega}_{r}^{*} \\
-\frac{1}{J} \dot{T}_{l}
\end{array}\right)
$$

where $\dot{\omega}_{r}^{*}$ and $\dot{T}_{1}$ are viewed as disturbances. By ignoring the disturbances, it can be written as:

$\dot{\mathbf{x}}=\mathbf{A x}+\mathbf{b} u$

The derivation of a dynamically linearized model of the SRM drive is based on the small deviant operation. The steady-state average torque of SRM drive derived by means of co-energy method is written as[13]:

$$
T_{a v}=m \frac{N_{r}}{2 \pi} \frac{U_{s}^{2}}{\omega_{r}^{2}}\left(\theta_{o f f}-\theta_{0}\right)\left[\frac{\theta_{0}-\theta_{o n}}{L_{\text {min }}}-\frac{1}{2} \frac{\theta_{o f f}-\theta_{0}}{L_{\max }-L_{\min }}\right]
$$

Equation (5) states that the average torque is a function of the parameters $U_{s}, \theta_{\text {on }}, \theta_{\text {off }}$ and $\omega_{r}$. By totally differentiating the average torque $T_{\alpha v}$, it leads to:

$$
d T_{a v}=\frac{\partial T_{a v}}{\partial U_{*}} d U_{s}+\frac{\partial T_{a v}}{\partial \theta_{o n}} d \theta_{o n}+\frac{\partial T_{a v}}{\partial \theta_{o f f}} d \theta_{o f f}+\frac{\partial T_{a v}}{\partial \omega_{r}} d \omega_{r}
$$


In vicinity of the operating point or on small deviant condition, by replacing the differential $d(*)$ of $(6)$ with the difference $\Delta(*)$, a dynamically linearized torque equation is obtained as:

$\Delta T_{a v}=K_{u} \Delta U_{s}+K_{o n} \Delta \theta_{o n}+K_{o f f} \Delta \theta_{o f f}-K_{\omega} \Delta \omega_{r}$ (7)

where

$K_{u}=\frac{m N_{r} U_{s}}{\pi \omega_{r}^{2}}\left(\theta_{o f f}-\theta_{0}\right)\left[\frac{\theta_{0}-\theta_{o n}}{L_{\min }}-\frac{1}{2} \frac{\theta_{o f f}-\theta_{0}}{L_{\max }-L_{\min }}\right]$

$K_{o n}=-\frac{m N_{r} U_{s}^{2}}{2 \pi L_{\text {min }} \omega_{r}^{2}}\left(\theta_{o f f}-\theta_{0}\right)$

$K_{\text {off }}=\frac{m N_{r} U_{s}^{2}}{2 \pi \omega_{r}^{2}}\left[\frac{\theta_{0}-\theta_{o n}}{L_{\min }}-\frac{\theta_{\text {off }}-\theta_{0}}{L_{\max }-L_{\min }}\right]$

$K_{\omega}=\frac{m N_{r} U_{s}^{2}}{2 \pi \omega_{r}^{3}}\left(\theta_{o f f}-\theta_{0}\right)\left[\frac{\theta_{0}-\theta_{o n}}{L_{\min }}-\frac{1}{2} \frac{\theta_{o f f}-\theta_{0}}{L_{\max }-L_{\text {min }}}\right]$

The mechanical equation of (1) is similarly written as:

$\Delta T_{a v}=J \frac{d \Delta \omega_{r}}{d t}+B \Delta \omega_{r}+\Delta T_{l}$

When the angular position control (APC) [13] mode is fired and a phase is turned on, the control angular commands will not be responded until a rotor pitch angular time-delay $\tau$ which waits for the phase to be turned on again. Let $\Delta \theta_{o n}^{*}$ and $\Delta \theta_{o f f}^{*}$ be the command angular inputs, then:

$\Delta \theta_{o n}=\Delta \theta_{o n}^{*}(t-\tau)$

$\Delta \theta_{\text {off }}=\Delta \theta_{\text {off }}^{*}(t-\tau)$

where $\tau$ is the average angular response time-delay and normally about several milliseconds. Equations (7), (12), (13) and (14) compose the dynamically linearized model of SRM drive in the time-domain.

\section{FSMC FOR SRM DRIVES [12],[14]}

The FSMC system of a three phase SRM drive is shown in Fig.1 in which there are two control loops: inner current and outer speed loops. The input of FSMC is the difference between the speed reference and speed feedback, while the corresponding output is the torque reference $T_{e}^{*}$. The current reference $i_{r e f}=f\left(T_{e}^{*}, \theta\right)$ is formulated according to the nonlinear torque-angle characteristic of the SRM drive. The design of the corresponding SMC is therefore divided into two parts: one dealing with the current control and the other with the speed control. The current sliding surface is defined as:

$s_{i}=i-i_{\text {ref }}$

From (1) and (15), its derivative can be written as:

$\dot{s}_{i}=\frac{d i}{d t}=-\frac{K i \omega}{L(\theta)}+\frac{u_{i}}{L(\theta)}$
Accordingly, the following sliding mode condition:

$s_{i} \dot{s}_{i}<0$

can be achieved by manipulating the phase voltage as:

$u_{i}= \begin{cases}V_{d c} & s_{i}<0 \\ -V_{d c} & s_{i}>0 \\ K i \omega & s_{i}=0\end{cases}$

From (18), the condition (17) can be satisfied when the SRM is operating in CCC mode.

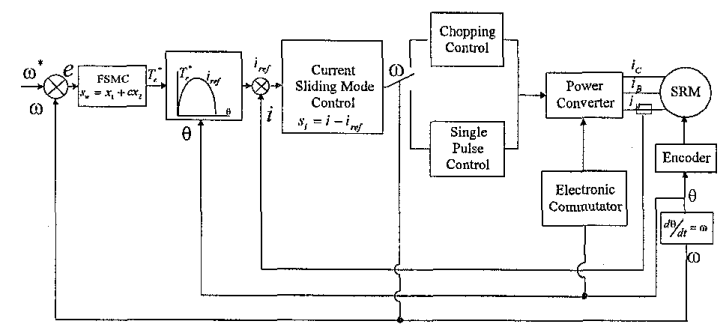

Fig. 1. SRM drive with FSMC.

The speed control is designed by defining a speed sliding surface as:

$s_{\mathrm{a}}=x_{1}+c x_{2}=\left(\begin{array}{ll}1 & c\end{array}\right)\left(\begin{array}{ll}x_{1} & x_{2}\end{array}\right)^{T}=\mathbf{c}^{T} \mathbf{x}$

where the dynamic behavior of the control system is determined by parameter $\mathbf{c}$. The sliding mode condition, $s_{\omega} \dot{s}_{\omega}<0$, is satisfied if a sliding mode control law is given by:

$u_{\omega}=\left\{\begin{array}{rr}200 & s_{\omega}<0 \\ u_{e q} & s_{\omega}=0 \\ -200 & s_{\omega}>0\end{array}\right.$

where

$u_{e q}=\left(J B-\frac{J}{c}\right) x_{2} \approx-\frac{J}{c} x_{2}=\frac{J}{c^{2}}\left(s_{\omega}-x_{1}\right)$

The basic configuration of a FLC comprises three principal parts: fuzzifier, rule base with inference engine, and defuzzifier. The main function of fuzzifier is to convert crisp data into fuzzy sets. Let $s \in S$ be an input variable in the universe of discourse $S . \widetilde{P}_{s} \in \widetilde{A}$, $\widetilde{N}_{s} \in \widetilde{A}$ and $\widetilde{Z}_{s} \in \widetilde{A}$ are three fuzzy subsets in the universe of discourse $\widetilde{A}=\left\{\widetilde{A}_{i}\right\}$ :

$\widetilde{P}_{s}: s$ is positive

$\widetilde{Z}_{s}: s$ is zero

$\tilde{N}_{s}: s$ is negative

As shown in Fig. 2 (a), these membership functions are continuous but not differentiable mappings and are written as:

$\mu_{s . p}(s)= \begin{cases}1, & \text { if } \quad s>\delta_{s} \\ s / \delta_{s}, & \text { if } \quad 0<s \leq \delta_{s} \\ 0, & \text { if } s<0\end{cases}$ 


$$
\begin{aligned}
& \mu_{s, n}(s)=\left\{\begin{array}{lll}
-s / \delta_{s}, & \text { if } & -\delta_{s}<s \leq 0 \\
1, & \text { if } & s \leq-\delta_{s} \\
0, & \text { if } & s>0
\end{array}\right. \\
& \mu_{s: z}(s)=\left\{\begin{array}{lll}
1-s / \delta_{s}, & \text { if } & 0<s \leq \delta_{s} \\
1+s / \delta_{s}, & \text { if } & -\delta_{s}<s \leq 0 \\
0, & \text { if } & |s|>\delta_{s}
\end{array}\right.
\end{aligned}
$$
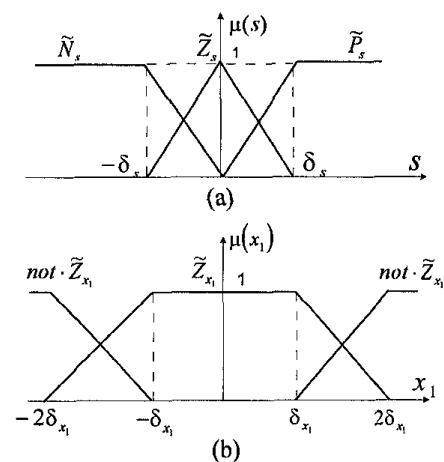

Fig. 2. Membership functions

Similarly another variable $x_{1}$ is defined in Fig. 2 (b). $\widetilde{A}_{i}$ and $\widetilde{B}_{i}$ are the fuzzy subsets of $\widetilde{A}$ and $\widetilde{B}$ over variables $s$ and $x_{1}$ respectively. $U$ is a bounded universe for control action $u$, and $U_{i}$ is the subset of $U$. The fuzzy rules containing two inputs and one output can be written in the first-order Sugeno fuzzy model [2] to obtain a nonlinear state feedback control gain:

$R_{1}:$ if $s$ is $\widetilde{P}_{s}$ then $u_{1}=-\alpha$

$R_{2}:$ if $s$ is $\widetilde{N}_{*}$ then $u_{2}=\alpha$

$R_{3}:$ if $s$ is $\widetilde{Z}_{s}$ and $x_{1}$ is not $\widetilde{Z}_{x_{1}}$ then $u_{3}=u_{e q 1}$

$R_{4}:$ if $s$ is $\widetilde{Z}_{x}$ and $x_{1}$ is $\widetilde{Z}_{x_{1}}$ then $u_{4}=u_{e q 2}$

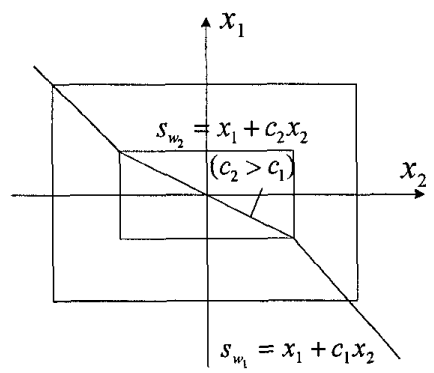

Fig. 3. Switching functions on a phase plane

In above fuzzy relations, two different control actions, $u_{e q 1}$ and $u_{e q 2}$, are used in $R_{3}$ and $R_{4}$ respectively. These control actions have different slopes $c_{1}$ and $c_{2}$ of switching lines $s_{0}=x_{1}+c_{i} x_{2}, i=1,2$. As shown in Fig. 3, when the state point is close to the origin of the phase plane, or $s \rightarrow 0$ and $x_{1} \rightarrow 0$, the rule $R_{4}$ is used to reduce overshoot and speed up the response time of system with a more gentle slope of switching line.

The center of gravity method is employed for the defuzzification of the output action $u$ [3], [4]:

$u=\sum_{i=1}^{l} \zeta_{i} u_{i} / \sum_{i=1}^{l} \zeta_{i}$

where $\zeta_{i}$ is obtained that:

$$
\zeta_{i}=\min \left(\mu_{\tilde{A}_{i}}(s), \mu_{\tilde{B}_{i}}\left(x_{1}\right)\right)
$$

where $\mu_{\tilde{A}_{i}}(s)$ and $\mu_{\tilde{B}_{i}}\left(x_{1}\right)$ are the grades of memberships of $x_{1}$ to $\widetilde{A}_{i}$ and $s$ to $\widetilde{B}_{i}$ respectively.

\section{TRANSFER FUNCTIONS OF SRM DRIVE SYSTEM}

The Laplace transforms of the dynamically linearized model, (7), (12), (13) and (14), are obtained as:

$$
\left\{\begin{array}{l}
\Delta T_{o v}(s)=K_{u} \Delta U_{s}(s)+K_{o n} \Delta \theta_{o n}(s)+K_{o f f} \Delta \theta_{o f f}(s)-K_{\omega} \Delta \omega_{r}(s) \\
\Delta T_{o v}(s)=J s \Delta \omega_{r}(s)+B \Delta \omega_{r}+\Delta T_{i}(s) \\
\Delta \theta_{o n}(s)=\Delta \theta_{o n}^{*}(s) e^{-\tau s} \\
\Delta \theta_{o f f}(s)=\Delta \theta_{o f f}^{*}(s) e^{-\tau s}
\end{array}\right.
$$

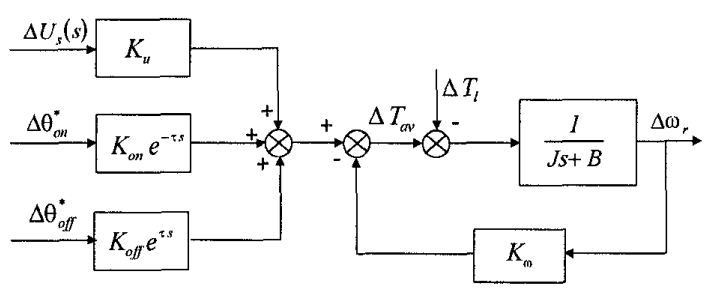

Fig. 4. Transfer function model of SRM drive.

Equation (27) illustrated by a block diagram as Fig. 4 depicts that there are three inputs, $\Delta U_{s}, \Delta \theta_{o n}^{*}$ and $\Delta \theta_{\text {off }}^{*}$. Correspondingly there are three different control outputs for the SRM control drive.

4.1 Transfer functions of power converter

1) CCC mode

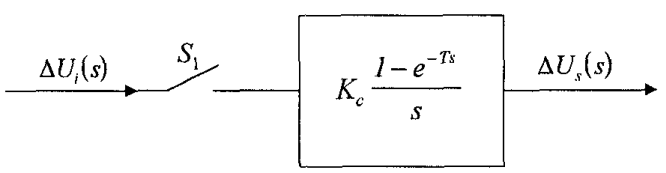

Fig. 5. CCC mode configuration.

$\Delta U_{s}$ represents input of the power converter in the $\mathrm{CCC}$ mode. If the current chopping is regarded as a process of sampling the output signal of the FSMC regulator with a gain $K_{c}$ and sampling period $T$, then configuration of SRM drive can be illustrated as Fig. 5 
in which $\Delta U_{i}(s)$ is the chopping signal of FSMC regulator output and $T$ is the sampling period determined by the chopping frequency.

2) APC mode

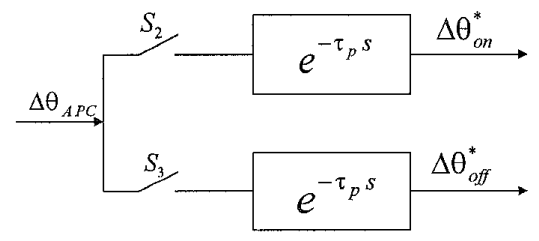

Fig. 6. APC mode configuration.

The command angular input, $\Delta \theta_{A P C}$, is passed through the power converter without other changes except a time-delay $\tau_{p}$, therefore the block diagram of control configuration is shown as Fig. 6. Similarly $\Delta \theta_{A P C}$ is the angular position signal of FSMC controller output.

\subsection{Transfer functions of speed and feedback elements}

From (25), the FSMC action signal $u$ is given as follows:

$u=\frac{\sum_{i=1}^{4} \mu_{R_{i}} u^{i}}{\sum_{i=1}^{4} \mu_{R_{i}}}=a_{1} x_{1}+a_{2} x_{2}=a_{1} x_{1}+a_{2} \dot{x}_{1}$

and the Laplace transform of (28) is written as:

$U(s)=\left(a_{1}+a_{2} s\right) X_{1}(s)$

From (2) the corresponding actuating torque $T_{e}^{*}$ is obtained as:

$T_{e}^{*}=\int_{0}^{t} \dot{T}_{e}^{*} d t=\int_{0}^{t} u d t$

Equation (30) demonstrates that there an integral action between control object and FSMC regulator. Considering (29), the Laplace transform of (30) yields:

$T_{e}^{*}(s)=\frac{1}{s} U(s)=\frac{a_{1}+a_{2} s}{s} X_{1}(s)=\left(a_{2}+\frac{a_{1}}{s}\right) X_{1}(s)$

The transfer function $G_{r}(s)$ from $x_{1}$ to $T_{e}^{*}$ is therefore obtained by:

$G_{r}(s)=\frac{T_{e}^{*}(s)}{X_{1}(s)}=a_{2}+\frac{a_{1}}{s}=K_{p}\left[1+\frac{1}{\left(T_{I} s\right)}\right]$

where $K_{p}=a_{2}$ is the proportional gain and $T_{I}=\frac{a_{2}}{a_{1}}$ is the integral time constant. Equation (32) states that the FSMC is equivalent to an adaptive PI regulator, whose parameters $a_{1}$ and $a_{2}$ can be adjusted to adapt the changing operation condition and to obtain the desired performance. Therefore, the FSMC demonstrates the powerful and robust control characteristics.
The speed measurement frequently adopted in the SRM drive is so-called the measuring frequency/period $(\mathrm{M} / \mathrm{T})$ method. The transfer function $\mathrm{G}_{\omega}$ of $\mathrm{M} / \mathrm{T}$ speed measurement feedback element is equivalently expressed by use of an inertial element:

$G_{\omega}=\frac{K_{n}}{1+T_{\omega} s}$

where $K_{n}$ is speed feedback gain and $T_{\omega}$ is speed feedback time constant.

4.3 Configuration and transfer functions of SRM drive

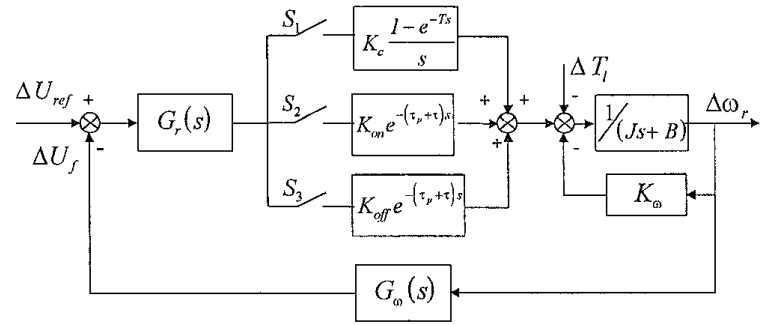

Fig. 7. Dynamically linearized configuration.

The dynamic-state configuration of the SRM drive system is shown in Fig. 7. The $S_{1} \sim S_{3}$ represent the switching of operation mode on different control condition which has correspondent transfer functions.

1) CCC mode ( $S_{1}$ is closed)

With the reference of Fig. 7, the system open-loop transfer function is given as follows:

$$
\begin{aligned}
G_{\mathrm{oc}}(s) & =G_{r}(s) K_{c} K_{u} \frac{1-e^{-T s}}{s} \frac{G_{\omega}(s)}{\left[J_{s} /\left(B+K_{\omega}\right)+1\right]\left[B+K_{\omega}\right]} \\
& =\frac{K_{o c}\left[1+T_{I} s\right]\left[1-e^{-T s}\right]}{T_{I} s^{2}\left(T_{\omega} s+1\right)\left(T_{M^{s}} s+1\right)}
\end{aligned}
$$

where $\quad K_{c c}=K_{p} K_{c} K_{u} K_{n} /\left(B+K_{\omega}\right) \quad$ and $T_{M}=J /\left(B+K_{\omega}\right)$.

The system closed-loop transfer function is:

$$
\begin{aligned}
G_{\mathrm{cc}}(s) & =\frac{G_{\mathrm{oc}}(s)}{1+G_{\mathrm{oc}}(s)} \\
= & \frac{K_{o c}\left(1+T_{I} s\right)\left(1-e^{-T s}\right)}{T_{I} s^{2}\left(T_{\mathrm{oc}} s+1\right)\left(T_{M} s+1\right)+K_{o c}\left(1+T_{I} s+T_{l} T_{D} s^{2}\right)\left(1-e^{-T s}\right)}
\end{aligned}
$$

The zero-order hold of (35) is approximately equivalent to an inertial element, that is:

$$
\frac{1-e^{-T s}}{s} \cong \frac{T}{1+s T}
$$


Substitution of (36) into (34) and approximation of two first-order elements with small time-constants, $T$ and $T_{\omega}$, by an equivalent inertial element, (34) becomes:

$G_{\mathrm{oc}}=\frac{K_{\mathrm{oc}}^{\prime}\left(1+T_{I} s\right)}{T_{I} s\left(T_{M} s+1\right)\left(T_{E} s+1\right)}$

where $K_{\mathrm{oc}}^{\prime}=K_{p} K_{c} K_{u} K_{n} T /\left(B+K_{\omega}\right)$ and $T_{E}=T+T_{\omega}$.

2) APC control mode ( $S_{2}$ is closed)

When $\theta_{o n}$ is control variable, a pure time-delay $e^{-\left(\tau_{p}+\tau\right) s}$ element exists in the system as shown in Fig. 7. Since the operation speed is beyond the base-speed $\omega_{b}$ to APC control mode, the time constant $\tau$ of the time-delay element is small and about several milliseconds normally. Hence the pure time-delay element can be approximated by use of an inertial element as:

$e^{-\left(\tau_{p}+\tau\right) s} \cong \frac{1}{1+\left(\tau_{p}+\tau\right) s}$

Combination of (38) with another small time constant inertial element (33), an equivalent element becomes:

$G_{\omega}(s) e^{-\left(\tau_{p}+\tau\right) s} \cong \frac{K_{n}}{T_{E} s+1}$

where $T_{E}=\tau_{p}+\tau+T_{\omega}$ is equivalent time constant. Therefore, the system open-loop transfer function is obtained as:

$$
\begin{aligned}
G_{o \theta_{o n}}(s) & =\frac{G_{r}(s) K_{o n} K_{n}}{\left(B+K_{\omega}\right)\left(T_{M} s+1\right)\left(T_{E} s+1\right)} \\
& =\frac{K_{o A}^{\prime}\left(1+T_{I} s\right)}{T_{I} s\left(T_{M} s+1\right)\left(T_{E} s+1\right)}
\end{aligned}
$$

where $K_{o A}^{\prime}=K_{o n} K_{n} K_{p} /\left(B+K_{\omega}\right)$, and its closed-loop transfer function is:

$$
G_{\mathrm{co}_{o n}}(s)=\frac{K_{o A}^{\prime}\left(1+T_{I} s\right)}{K_{O A}^{\prime}\left(1+T_{I} s\right)+T_{I} s\left(T_{M} s+1\right)\left(T_{E} s+1\right)}
$$

3) APC control mode ( $S_{2}$ and $S_{3}$ are closed at same time)

Both $\theta_{\text {on }}$ and $\theta_{\text {off }}$ are control variables. In a similar method of 2), the system open-loop transfer function is obtained as:

$$
\begin{aligned}
G_{\mathrm{OA}}(s) & =\frac{G_{r}(s)\left(K_{o n}+K_{o f f}\right) K_{n}}{\left(T_{M} s+1\right)\left(B+K_{\omega}\right)\left(T_{E} s+1\right)} \\
& =\frac{K_{o A}\left(1+T_{l} s\right)}{T_{I} s\left(T_{M} s+1\right)\left(T_{E} s+1\right)}
\end{aligned}
$$

where $K_{o A}=K_{p}\left(K_{o n}+K_{o f f}\right) K_{n} /\left(B+K_{\mathrm{\omega}}\right)$ and its closed-loop transfer function is:
$G_{\mathrm{cA}}(s)=\frac{K_{\mathrm{oA}}\left(1+T_{I} s\right)}{T_{I} s\left(T_{E} s+1\right)\left(T_{M} s+1\right)+K_{o A}\left(1+T_{I} s\right)}$

\section{SRM DRIVE SYSTEM OPERATION ANALYSIS}

\subsection{Stability criterion}

A minor-loop feedback is contained in the closedloop SRM drive system as shown in Fig. 7. The necessary condition of the closed-loop system stability is the minor-loop feedback to be stable. From Fig. 7, the pole of the minor-loop feedback is:

$p_{M}=-\left(B+K_{\omega}\right) / J$

then the stable condition $p_{M}<0$ of the minor-loop feedback results in:

$K_{\omega}>-B$

Since the value of the viscous friction coefficient $B$ is very small, it is clear from (11) and (45) that the SRM drive system will be stable provided that inequality:

$K_{\omega}>0$

is satisfied by properly determining control variables $\theta_{o n}$ and $\theta_{o f f}$. According to (11), the following inequality must be fulfilled to obtain a stable system operation:

$\left(\theta_{\text {off }}-\theta_{0}\right)\left[\frac{\theta_{0}-\theta_{o n}}{L_{\text {min }}}-\frac{1}{2} \frac{\theta_{o f f}-\theta_{0}}{L_{\text {max }}-L_{\text {min }}}\right]>0$

Since inequalities $\theta_{o f f}>\theta_{0}$ and $\theta_{0}>\theta_{o n}$ are tenable, then following inequality becomes the system stable criterion:

$\frac{\theta_{0}-\theta_{o n}}{L_{\min }}>\frac{1}{2} \frac{\theta_{o f f}-\theta_{0}}{L_{\max }-L_{\min }}$

The characteristics of variable parameter $K_{\omega}$ versus speed $\omega_{r}$ in APC mode is shown in Fig. 8.

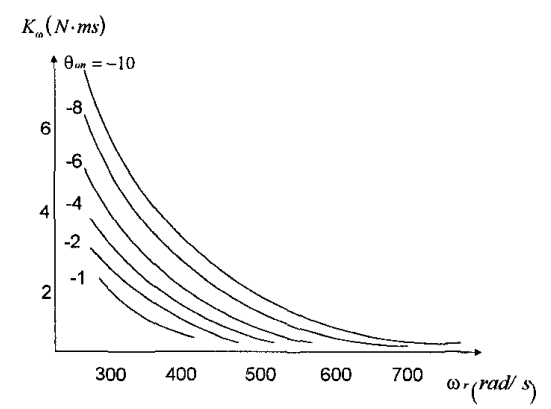

Fig. 8. $K_{\omega}$ vs. $\omega_{r}$ in APC mode $\left(\theta_{\text {off }}=12.5^{\circ}\right)$.

Although the SRM drive system has different control configuration and transfer function to different control 
mode, it is noted from (34), (40) and (42) that it can be expressed by a unified open-loop transfer function with the same construction but different parameter values, that is:

$$
G_{\text {op }}(s)=\frac{K\left(s-z_{1}\right)}{s\left(s-p_{1}\right)\left(s-p_{2}\right)}
$$

If the FSMC system has a real zero $z_{1}=-2$ and a pair of real poles, $p_{1}=-1 / T_{M}=-1$, $p_{2}=-1 / T_{E}=-5$, then the system open-loop transfer function (49) can be written as:

$G_{\mathrm{op}}=\frac{K(s+2)}{s(s+1)(s+5)}$

The root locus of the closed-loop SRM drive system of (50) is illustrated in Fig. 9. With the above-given zero and poles of the system indicated in Fig. 9, when open-loop gain parameter $K$ varies from 0 to $\infty$, the root locus of the closed-loop system is always located on the left-half plane, therefore the closed-loop system is stable to any value of gain parameter $K$.

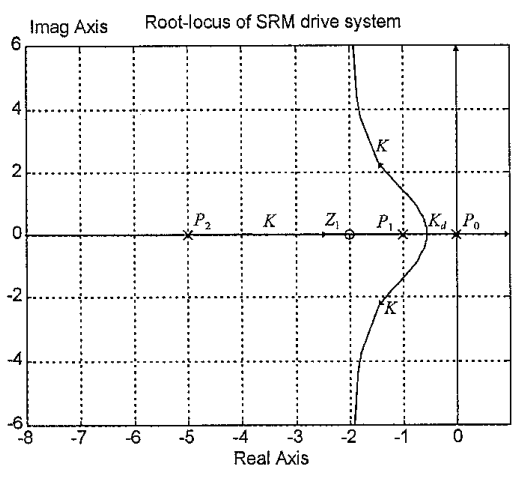

Fig. 9. The root locus of the closed-loop.

In general, the closed-loop system is stable provided that all open-loop zero $z_{1}$, and closed-loop poles, $p_{1}$ and $p_{2}$, lie on the left-half plane. Since $z_{1}$ and $p_{2}$ are inevitably negative, the sufficient and necessary condition for the system stability becomes:

$p_{1}=-1 / T_{M}=-\left(B+K_{\omega}\right) / J<0$

which is exactly (44). Therefore, provided that the angular control parameters $\theta_{\text {on }}$ and $\theta_{\text {off }}$ are properly chosen, inequalities (48) and (51) can be fulfilled and the whole SRM drive system is stable.

With the reference of Fig. 1, when the sliding mode existence condition is satisfied, a manipulated torque $T_{e}^{*}$ is inferred from the FSMC, then the actuating signal, $i_{\text {ref }}=f\left(T_{e}^{*}, \theta\right)$ for CCC mode or $\theta_{o n}^{*}=f\left(T_{e}^{*}, \omega_{r}\right)$ for APC mode, is given. To CCC mode with fixed $\theta_{\text {on }}$ and $\theta_{\text {off }}$, the stability criteria (46) and (48) are inevitably fulfilled. Whereas to APC mode, the com- mand $\theta_{o n}^{*}$ satisfies both of (46) and (48). Therefore the sliding mode condition guarantees the FSMC system stability.

\subsection{Steady-state error analysis}

With the reference of Fig. 7, if system is subjected to an input $\Delta U_{r e f}$, the steady-state error is defined as:

$e_{s s}(t)=\lim _{s \rightarrow 0} \frac{s \Delta U_{r e f}(s)}{1+G_{\mathrm{op}}(s)}$

Because the open-loop transfer function (50) of SRM drive system has an open-loop pole at the origin of complex plane, if the system is subjected to a unit-step input $\Delta U_{r e f}=\frac{1}{s}$, the system steady-error can be obtained by substituting the input into (52):

$e_{s s}(t)=\lim _{s \rightarrow 0} \frac{1}{1+G_{\mathrm{op}}(s)}=0$

To a unit-ramp input $\Delta U_{r e f}=\frac{1}{s^{2}}$, the system steadyerror is given as:

$e_{s s}=\lim _{s \rightarrow 0} \frac{s \Delta U_{r e f}(s)}{1+G_{\mathrm{op}}(s)}=\frac{1}{K}$

According to (53) and (54), the SRM drive system with FSMC is said to be a type 1 system whose unit-step steady-state error is zero. The steady-state velocity error of the system is in inverse proportion to the gain parameter $K$ or $e_{s s} \propto K^{-1}$. If system is given a steadystate velocity error requirement, the tolerance of the gain parameter $K$ can be obtained from the system root locus shown in Fig. 9. It is seen from the open-loop transfer functions of (34), (40) and (42) that the gain parameter $K$ is manipulated by the signal $u$ and the slope $c$ of the sliding surface $s$ in FSMC scheme.

\section{SYSTEM SIMULATION AND HARDWARE IMPLEMENTATION}

\subsection{Computer simulation}

\begin{tabular}{ll}
\multicolumn{2}{c}{ Table 1. System parameters of SRM drive. } \\
\hline Power & $4 \mathrm{~kW}$ \\
Phase & 3 \\
Stator poles & 12 \\
Rotor poles & 8 \\
DC voltage & $240 \mathrm{~V}$ \\
Rated current & $40 \mathrm{~A}$ \\
Rated torque & $12.8 \mathrm{Nm}$ \\
Stator resistance & $0.3 \Omega$ \\
Moment of inertia & $0.031 \mathrm{Nms}$ \\
Viscous friction coefficient & $0.0012 \mathrm{Nms}^{2}$ \\
\hline
\end{tabular}

As shown in Table 1, the parameters of a practical 3phase 4kW SRM drive are used for computer simulation. During the simulation, the applied voltage and line current are detected without time delay, the dead 
time and turn on/off time of IGBTs are taken into account, and the current control is performed with current hysteresis controlled PWM.

Various simulations are performed under different initial states, speed references, load disturbances and system parameter deviations so that the fast tracking capability and robustness of the drive system can be testified.

Fig.10 shows the traces of the instantaneous torque developed by using FSMC and the conventional SMC under the same working conditions. The result demonstrates that the FSMC is stable and robust.
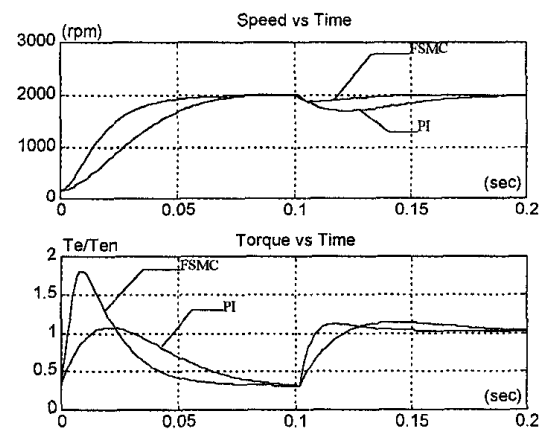

Fig. 10. Speed and torque responses of FSMC and PI with initial $5 \mathrm{Nm}$ and then $17 \mathrm{Nm}$ at $0.1 \mathrm{~s}$.

\subsection{Hardware implementation}

The practical realization of the proposed FSMC algorithm is based on the Motorola MC68HC16Z1 microcontroller as shown in Fig. 11. The FSMC controller deals with the phase current chopping and switching pattern computations with a sampling time of $10 \mathrm{~ms}$.

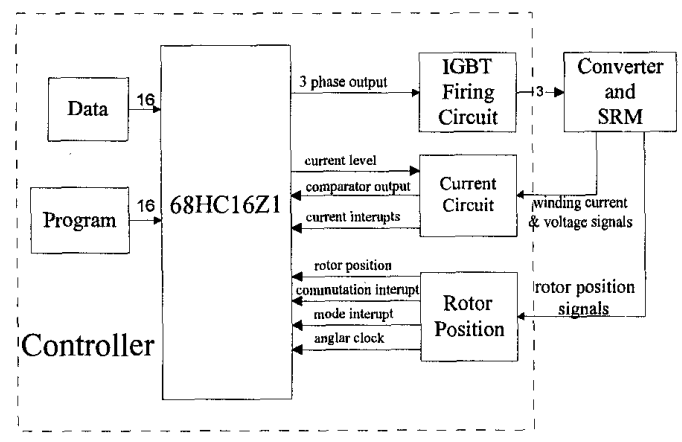

Fig. 11. Hardware schematic of FSMC for SRM drive.

\section{ACKNOWLEDGMENT}

This work was supported and funded in part by the Committee on Research and Conference Grants, the University of Hong Kong.

\section{CONCLUSIONS}

The application of FSMC to a SRM drive has been proposed and implemented. The transfer functions of SRM drive system are derived by its small deviation model. From the characteristics of the transfer functions, it is concluded that the FSMC speed regulator can be regarded as an adaptive PI regulator. The stability analysis dedicates that the stability condition based on the transfer functions is consistent with the sliding mode existence condition $\dot{s} s<0$. The steady-state error analysis states that the SRM drive system with FSMC is a type 1 system with zero error of unit-step input. Computer simulation results verify that the proposed FSMC can effectively compensate the nonlinear torque characteristics of the SRM drive. The FSMC is insensitive to the system parameter uncertainty and variations, and is robust against external disturbances.

\section{REFERENCES}

[1] P. Guillemin, "Fuzzy logic applied to motor control," IEEE Transactions on Industry Applications, Vol. 32, No. 1, pp. 51 $56,1996$.

[2] T. Takagi and M. Sugeno, "Fuzzy identification of systems and its applications to modeling and control," IEEE Transactions on Systems, Man, and Cybernetics, Vol. 15, No. 1, pp. 116132,1985

[3] A. Kandel and G. Lagholz, "Fuzzy control systems," CRC Press, pp. 227-242, 1993.

[4] S.G. Tzafestas and A.N. Venetsanopoulos, "Fuzzy reasoning in information, decision and control systems," Kluwer Academic Publishers, pp. 277-328, 1994.

[5] C. Rossi and A. Toniell, "Feedback linearizing and sliding mode control of a variable reluctance motor," International Journal of Control, Vol. 60, No. 4, pp. 543-568, 1994.

[6] C.B. Buja, R. Menis and M. Valla, "Variable structure control of an SRM drive," IEEE Transactions on Industrial Electronics, Vol. 40, No. 1, pp. 56-63, 1993.

[7] V.I. Utkin, "Variable structure systems with sliding modes," IEEE Transactions on Automatic Control, Vol. 22, No. 2, pp. 212-218, 1977.

[8] J.J. Slotine, "Sliding controller design for non-linear systems," International Journal of Control, Vol. 40, No. 2, pp. 421434, 1984 .

[9] S. Bolognani, E. Ognibeni and M. Zigliotto, "Sliding mode control of the energy recovery chopper in a C-dump switched reluctance motor drive," IEEE Transactions on Industry Applications, Vol. 29, No. 1, pp. 181-186, 1993.

[10] A. Ishigame and T. Furukawa, "Sliding mode controller design based on fuzzy inference for nonlinear systems," IEEE Transactions on Industrial Electronics, Vol. 40, No. 1, pp. 64-70, 1993.

[11] J.C. Wu and T.S. Liu, "A sliding-mode approach to fuzzy control design," IEEE Transactions on Control System Technology, Vol. 4, No. 2, pp. 141-151, 1996.

[12] C.C. Chan, Y.J. Zhan and K.T. Chau, "Fuzzy variable structure control of switched reluctance motor drive for EVs," Proceedings of International Electric Vehicle Symposium, pp. 573$578,1996$.

[13] T.J.E. Miller, "Converter volt-ampere requirements of the switched reluctance motor drive," IEEE Transactions on Industry Applications, Vol. 21, No. 5, pp. 1136-1144, 1985.

[14] Y.J. Zhan, "A High Performance Switched Reluctance Motor Drive for Electric Vehicles," Ph.D. thesis, 1998. 